\author{
定向合成高效捕获 $\mathrm{CO}_{2}$ 的新型多孔芳香材料 \\ 王维 ${ }^{a}$ 间卓君 ${ }^{b}$ 元野 ${ }^{b}$ 孙福兴 ${ }^{b}$ \\ 赵明*, 任浩*, $b$ 朱山 ${ }^{b}$ \\ ( ${ }^{a}$ 吉林大学材料科学与工程学院 长春 130022)

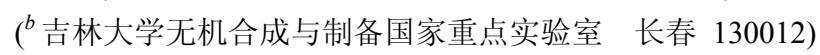

\begin{abstract}
摘要 通过简单的离子热法, 以四(4-氧基联苯基)硅烷作为四面体基块, 将其与无水氯化锌在充满氩气气氛的手套箱 中充分研磨后密封, 分别以 400 和 $550{ }^{\circ} \mathrm{C}$ 的反应温度合成了新型多孔芳香骨架材料(PAF-51), 得到 PAF-51-1 $\left(400{ }^{\circ} \mathrm{C}\right.$ 条 件下)与 PAF-51-2 (550 ${ }^{\circ} \mathrm{C}$ 条件下)的比表面积分别为 720 和 $557 \mathrm{~m}^{2} \cdot \mathrm{g}^{-1}$ (BET). 与 $\mathrm{CH}_{4}$ 和 $\mathrm{N}_{2}$ 对比, 该材料对 $\mathrm{CO}_{2}$ 具有极 好的选择性吸附能力. $273 \mathrm{~K}$ 条件下, $\mathrm{CO}_{2} / \mathrm{N}_{2}$ 分离指数最高可达 $52.2, \mathrm{CO}_{2} / \mathrm{CH}_{4}$ 分离指数也达到 10.3 , 这一性质极有可能 使得 PAF-51 成为捕获 $\mathrm{CO}_{2}$ 理想材料, 并对再生能源具有潜在的应用.
\end{abstract}

关键词 离子热反应; 多孔芳香材料; $\mathrm{CO}_{2}$ 选择性吸附; 富氮骨架

\title{
Target Synthesis of a Novel Porous Aromatic Framework for Efficient $\mathrm{CO}_{2}$ Capture
}

\author{
Wang, Wei ${ }^{a} \quad$ Yan, Zhuojun $^{b} \quad{\text { Yuan, } \text { Ye }^{b} \quad \text { Sun, Fuxing }}^{b}$ \\ Zhao, Ming*,a Ren, Hao ${ }^{*, b}$ Zhu, Guangshan ${ }^{b}$ \\ $\left({ }^{a}\right.$ Jilin University Key Laboratory of Automobile Materials, Ministry of Education, and School of Materials Science and \\ Engineering, Jilin University, Changchun 130022) \\ $\left({ }^{b}\right.$ Jilin University State Key Laboratory of Inorganic Synthesis \& Preparative Chemistry, Jilin University, \\ Changchun 130012)
}

Abstract In this report, novel porous aromatic frameworks (PAF-51) have been synthesized using tetrahedral units in the presence of zinc chloride by a facile ionothermal method. PAF-51-1 and PAF-51-2 displayed large surface areas as 720 and $557 \mathrm{~m}^{2} \cdot \mathrm{g}^{-1}$ (BET) respectively. Selectivity of $\mathrm{CO}_{2} / \mathrm{N}_{2}$ is as high as 52.2 at $273 \mathrm{~K}$ and $\mathrm{CO}_{2} / \mathrm{CH}_{4}$ can reach 10.3 at $273 \mathrm{~K}$. The properties make it a promise as the ideal candidate to capture $\mathrm{CO}_{2}$ and can be used for clean and renewable energy which still needs further study. FTIR spectra of initial monomers and final products gave us information of the reaction process. The absence of cyanogroups $\left(2230 \mathrm{~cm}^{-1}\right)$ of the TCDPSi can verify the completion of the trimerization reaction. The appearance of breathing vibration bands of triazine ring $\left(1560 \sim 1520\right.$ and $\left.1480 \sim 1350 \mathrm{~cm}^{-1}\right)$ and out-of-plane flexural vibration $(860 \sim$ $736 \mathrm{~cm}^{-1}$ ) indicate the formation of polymeric networks. Thermogravimetric analysis (TGA) under atmosphere of the two samples revealed a high thermal stability up to 415 (PAF-51-1) and $475{ }^{\circ} \mathrm{C}$ (PAF-51-2). Furthermore, these materials are all insoluble after long-period stirring in most common solvents, such as $1 \mathrm{~mol} / \mathrm{L} \mathrm{HCl}$, acetone, tetrahydrofuran (THF), $\mathrm{N}, \mathrm{N}$-dimethylformamide (DMF), $\mathrm{CHCl}_{3}$, etc., showing high chemical stability. FTIR spectra of PAF-51 before stirring in these solvents $\left(\mathrm{HCl}\right.$, acetone, THF, DMF, and $\mathrm{CHCl}_{3}$ etc.) and after stirring in solvents were barely changed. Powder X-ray diffraction (PXRD) of these PAFs revealed no distinct diffraction peaks, indicating that these polymers are composed of an amorphous network. Scanning electron microscopy (SEM) analyses display that the polymers consist of aggregated irregular lumps with nanometer dimensions. Transmission electron microscopy (TEM) investigations show their clear porous textures and agree on the amorphous structures. The surface areas of PAF-51-1 and PAF-51-2 are 720 and $557 \mathrm{~m}^{2} \cdot \mathrm{g}^{-1}$ on BET model respectively. At low relative pressure, inflection point of the isotherm of PAF-51-2 appears much earlier than PAF-51-1, which might be a suggestion of the distribution of micropores in the product. Indeed, pore size distribution calculated from nonlocal density functional theory (NLDFT) using the model of carbon as an adsorbent also demonstrates this. The pore size distribution of PAF-51-1 mainly exists in the region between 2 20 A. Although pore size distribution of PAF-51-2 also mostly exists in the microporous region, less distribution than that in PAF-51-1, and more distribution can be found in mesoporous region between $20 \sim 500 \AA$. The selectivity of PAF-51-1 reaches 34.1 which was calculated from the initial slopes of the $\mathrm{CO}_{2}$ and $\mathrm{N}_{2}$ adsorption isotherms. Much more remarkably, the selectivity at room temperature (298 K) decreases very little (from 34.1 to 32.4 ), which is better than many other porous materials with higher surface area and $\mathrm{CO}_{2}$

*E-mail: zhaoming@jlu.edu.cn; renhao@jlu.edu.cn

Received February 20, 2014; published April 13, 2014.

Supporting information for this article is available free of charge via the Internet at http://sioc-journal.cn.

Project supported by the National Natural Science Foundation of China (No. 20831002), Major International (Regional) Joint Research Project (No. 21120102034), National Key Basic Research and Development Program (No. 2010CB631001).

项目受国家自然科学基金(No. 20831002)、重大国际(地区)合作研究(No. 21120102034)、国家重点基础研究发展计划(No. 2010CB631001)资助. 
uptakes. Remarkably, the selectivity of PAF-51-2 is much higher than PAF-51-1 (52.2 at $273 \mathrm{~K}$ and 43.6 at $298 \mathrm{~K}$ ). Likewise, the selective adsorption of $\mathrm{CO}_{2}$ to $\mathrm{CH}_{4}$ can reach 10.3 at $273 \mathrm{~K}$.

Keywords ionothermal reaction; porous aromatic framework; $\mathrm{CO}_{2}$ adsorption separation; nitrogen-rich framework

\section{1 引言}

众所周知, $\mathrm{CO}_{2}$ 是导致全球变暖的温室气体中最重 要组成部分 ${ }^{[1]}$. 化石燃料以及一些石油衍生物在日常生 活中越来越广泛地应用, 大量的燃烧废气使空气中的 $\mathrm{CO}_{2}$ 含量加倍增长. 受其影响, 全球大气环流产生变化, 气候带向极地扩展, 引起了气候异常进而导致多种自然 灾害的发生 ${ }^{[2]}$. 此外, 气温升高还会引起和加剧传染病 的传播. 因此, 对大气中的 $\mathrm{CO}_{2}$ 进行捕获和分离是十分 必要的. 目前, 广泛使用有机胺溶液 ${ }^{[3]}$ 作吸附剂来吸收 工业生产过程中产生的 $\mathrm{CO}_{2}$, 这一方法在化石燃料发电 厂等处获得极为广泛的应用. 然而该方法带来的诸如经 济负担严重、极易造成设备腐蚀 ${ }^{[4]}$ 等缺点, 一直难以解 决.

作为代替有机胺溶液的材料, 多孔固体材料如硅 胶, 活性炭 ${ }^{[5]}$, 微介孔无机分子篎, 金属有机骨架 $\mathrm{MOFs}^{[6 \sim 8]}$, 多孔有机骨架聚合物等以物理吸附的方式 吸收 $\mathrm{CO}_{2}$ 吸引了众多材料学家的关注. 这些材料改进了 以胺类为主的化学吸附过程的一些缺点, 常温常压条件 下，具有高比表面积的有机多孔材料展现了显著的 $\mathrm{CO}_{2}$ 捕获及选择性吸附能力. 然而, 它们的合成过程复杂, 材料孔道与 $\mathrm{CO}_{2}$ 分子间缺少强相互作用, 稳定性也通常 较差, 因此开发制备出一系列合成方法简单并且具有高 稳定性的新型材料十分必要.

多孔有机骨架聚合物包括: 高交联聚合物及固有微 孔聚合物 HCPs/PIMs ${ }^{[9 \sim 11]}$, 共轭微孔聚合物 CMP ${ }^{[12]}$, 共价三嗪结构 $\mathrm{CTFS}^{[13 \sim 15]}$, 多孔聚合网状结构 $\mathrm{PPNs}^{[16,17]}$ ，以及由有机共轭片段构成的多孔芳香骨架 化合物 $\mathrm{PAFs}^{[18 \sim 21]}$ 等. 其中 PAFs 具有低骨架密度, 高 比表面积和高稳定性等特点, 使其在气体的捕获和分离 过程中, 能够获得较高的单位质量吸附率. 为了探索多 孔有机聚合物对 $\mathrm{CO}_{2}$ 的捕获性能, 我们设计合成了一种 新型的 PAFs 材料(PAF-51), 如图 1 所展示出的合成路 线. 该材料由四面体 TCDPSi 作为建筑基块, 通过离子 热法 ${ }^{[22]}$ 制备得到 PAF-51 材料. 其中含氧基的单体 TCDPSi 通过㲵基自聚反应形成三角雉结构的三嗪环, 其富氮结构能够富集电子, 增强骨架结构与 $\mathrm{CO}_{2}$ 分子间 相互作用 ${ }^{[23]}$, 因此对 $\mathrm{CO}_{2}$ 气体具有显著的吸附和选择能 力. 同时, 我们选择这样一个刚性单体, 也有利于骨架 的延伸, 以达到减少结构穿插的目的. 在这个反应中, 我们选择了不同的反应温度获得了不同孔径尺寸的材 料, 进而探究其对 $\mathrm{CO}_{2}$ 气体吸附和选择能力的影响.


图 1 PAF-51 的合成路线

Figure 1 The synthesis strategy of PAF-51

(a) a triazine ring $\left(\mathrm{C}_{3} \mathrm{~N}_{3}\right)$ formed via $\mathrm{ZnCl}_{2}$ mediated nitrile cyclotrimerization; (b) TCDPSi as the tetrahedral building unit and the possible fragment of PAF-51

\section{2 结果与讨论}

\section{1 红外光谱分析}

为了探索 PAF-51 材料的基本结构, 我们对其进行 了红外光谱分析. 如图 $2 \mathrm{a}$, 能够观察到反应单体中的 $\mathrm{CN}$ 峰 $\left(2230 \mathrm{~cm}^{-1}\right)$ 在产物中完全消失, 这一结果可以初 步确定产物已经按照预想的反应发生了聚合，且聚合程 度完全. 在图 $2 b$ 中, 位于三嗪环呼吸振动带 $(1560 \sim$ 1520 及 $\left.1480 \sim 1350 \mathrm{~cm}^{-1}\right)$ 和面外弯曲振动 $(860 \sim 736$ $\mathrm{cm}^{-1}$ )的特征峰，表明了聚合物中三嗪环的存在. 此外, 可以观察到 PAF-51-1 材料在苯环骨架振动带(1625 
$1580 \mathrm{~cm}^{-1}$ ) 有明显的吸收峰, 但 PAF-51-2 中却较弱甚至 难以观察到, 这在一定程度上证明了 PAF-51-2 有可能 在反应过程中经历了 PAF-51-1 的结构后, 又发生了局 部结构的坍塌.

(a)

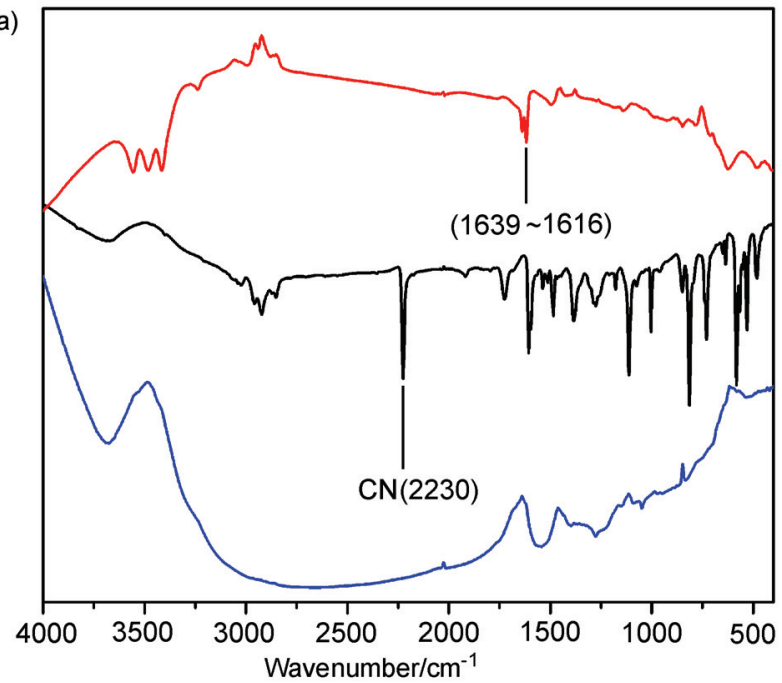

(b)

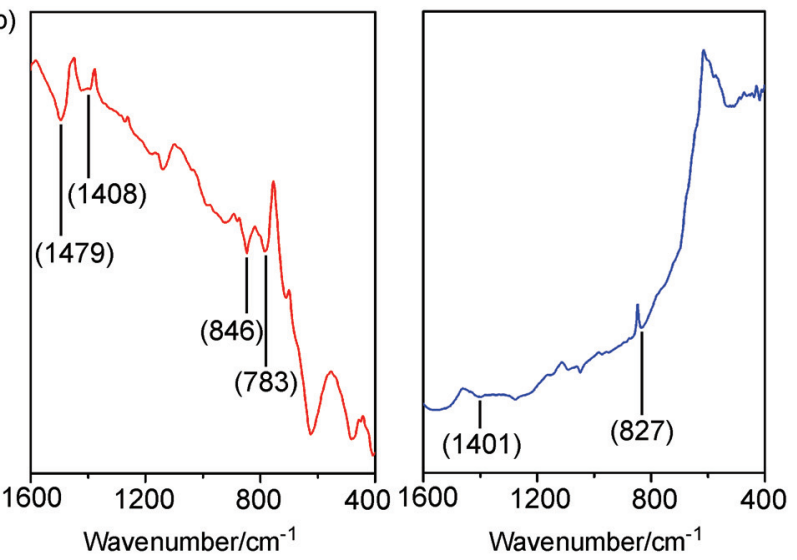

图 2 (a) 聚合物的红外图谱 PAF-51-1(红色曲线), PAF-51-2(蓝色曲 线), TCDPSi 单体(黑色曲线). (b)分别截取聚合物部分红外图谱分析结 构中三嗪环的存在

Figure 2 (a) FTIR spectra of PAF-51-1 (red), PAF-51-2 (blue) and TCDPSi (black); (b) Details of PAF-51-1 (red) and PF-51-2 (blue)

\section{2 电镜分析及粉末 X-ray 衍射分析}

通过对 PAF-51 材料进行粉末 X 射线衍射表征, 具 体分析了聚合物的结晶性. 如图 3 所示, 结果表明该物 质并无明显的长程有序结构. 我们对这种无定形材料的 形貌进行进一步表征, 如图 4a, 图 4b 所示, 在扫描电镜 图片中我们可以看到该类材料为微米级不规则块状聚 合物. 通过透射电镜分析(图 4c, 图 4d), 我们进一步确 定了该材料的无定形结构.

\section{3 稳定性能分析}

PAF-51-1 及 PAF-51-2 的热稳定性由热重分析仪在 空气环境下测定. 如图 5 所示, PAF-51-1 保持稳定质量 高达 $415{ }^{\circ} \mathrm{C}$. 相似的是, PAF-51-2 加热到 $475{ }^{\circ} \mathrm{C}$ 仅有较

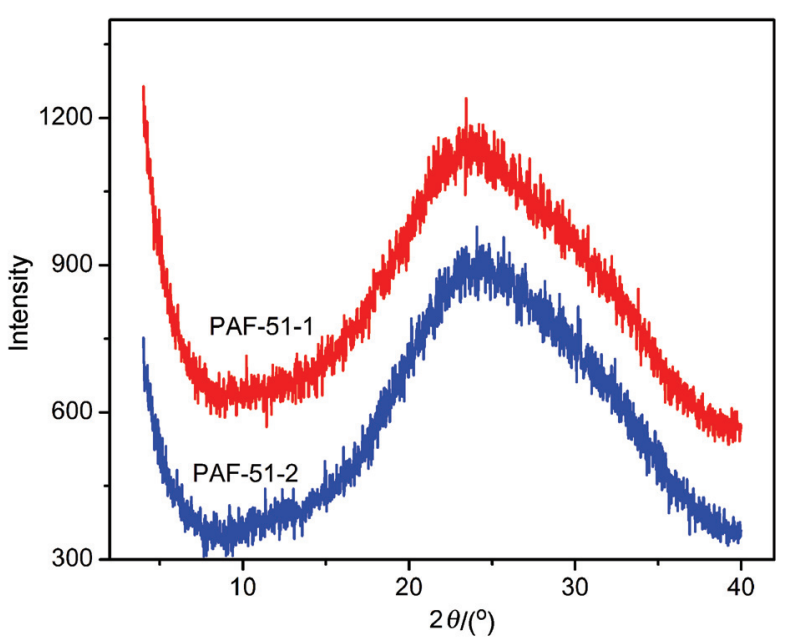

图 3 PAF-51-1(红色曲线)和 PAF-51-2(蓝色曲线)的粉末 X 射线衍射 Figure 3 The PXRD patterns of PAF-51-1 (red) and PAF-51-2 (blue)

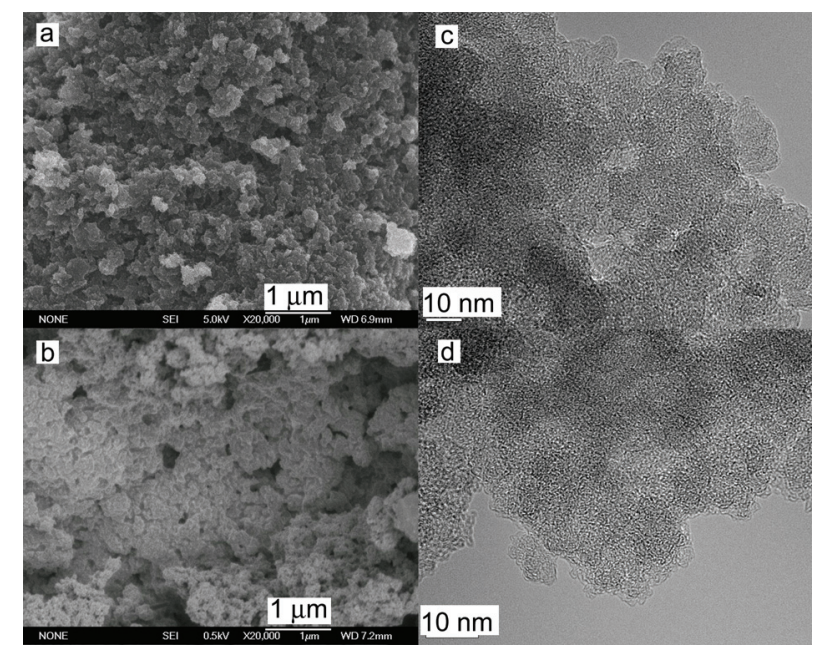

图 4 (a) PAF-51-1 的扫描电镜图谱; (b) PAF-51-2 的扫描电镜图谱;(c) PAF-51-1 的透射电镜图谱; (d) PAF-51-2 的透射电镜图谱

Figure 4 (a) SEM image of PAF-51-1; (b) SEM image of PAF-51-2; (c) HRTEM image of PAF-51-1; (d) HRTEM image of PAF-51-2

少的失重. 这表明该类材料具有极高的热稳定性. 此外, PAF-51 系列材料也展现出了非常好的溶剂稳定性, PAF-51 材料在 $1 \mathrm{~mol} / \mathrm{L}$ 盐酸、丙酮、四氢呋喃、 $N, N^{\prime}-$ 二甲基甲酰胺、氯仿等常规溶剂中经过长周期的搅拌, 均不会发生溶解. 我们将 PAF-51 材料依次先后投入上 述溶剂中, 每种溶剂中摚拌 $2 \mathrm{~d}$, 最终所得产物再次进 行红外光谱分析(图 S2), 测试结果与搅拌前并无明显差 异.

\section{4 孔道性质分析}

为了研究 PAF-51 的孔道性质, 我们测定了它们在 $77 \mathrm{~K}$ 时的 $\mathrm{N}_{2}$ 吸附. 测定前样品进行了一系列预处理过 程: 首先, 样品用去离子水充分洗涤, 以除去 $\mathrm{ZnCl}_{2}$. 其 次, 所得沉淀加入 $1 \mathrm{~mol} / \mathrm{L}$ 盐酸中进一步提纯. 然后, 用 无水四氢呋喃充分交换产物中的客体水分子. 最后，产 


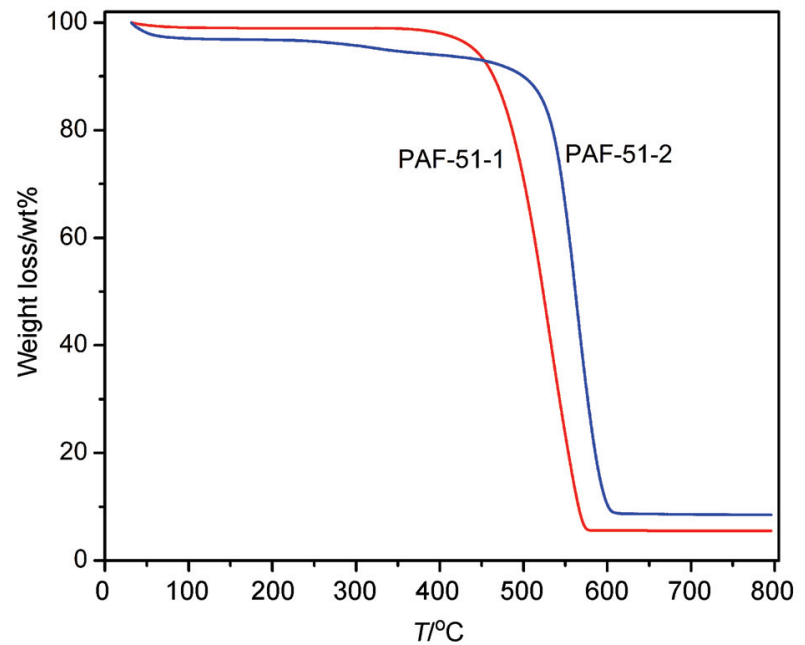

图 5 红色的曲线为 PAF-51-1 的热重曲线; 蓝色的曲线为 PAF-51-2 的热重曲线

Figure 5 TGA plots for two polymer networks PAF-51-1 (red) and PAF-51-2 (blue) at air condition with the rate of $5{ }^{\circ} \mathrm{C} \cdot \mathrm{min}^{-1}$

物在 $100{ }^{\circ} \mathrm{C}$ 高真空的环境下干燥 $6 \mathrm{~h}$.

如图 6 所示, 在 $P / P_{0}=1 \times 10^{-5} \sim 1 \times 10^{-2}$ 的极低压 区，吸附等温线急剧上升，显示了聚合物强烈的 $\mathrm{N}_{2}$ 吸附 性质. 等温线在高压区有微小的滞后环, 这一结果可能 是由于材料在反应中发生的结构穿插以及孔道的局部 坍塌造成的. 由不同反应温度得到的 PAF-51-1 和 PAF-51-2, 通过 $\mathrm{N}_{2}$ 吸附表征, 应用 BET 模型得出其比表 面积差异很大, PAF-51-1 为 $720 \mathrm{~m}^{2} \cdot \mathrm{g}^{-1}$, PAF-51-2 为 557 $\mathrm{m}^{2} \cdot \mathrm{g}^{-1}$, 以碳为模型, 应用非局部密度泛函理论计算得 出其孔径分布也有着明显差别. 如图 6 内小图所示, 反 应温度为 $400{ }^{\circ} \mathrm{C}$ 的 PAF-51-1 孔径分布主要在 2 20 $\AA$,

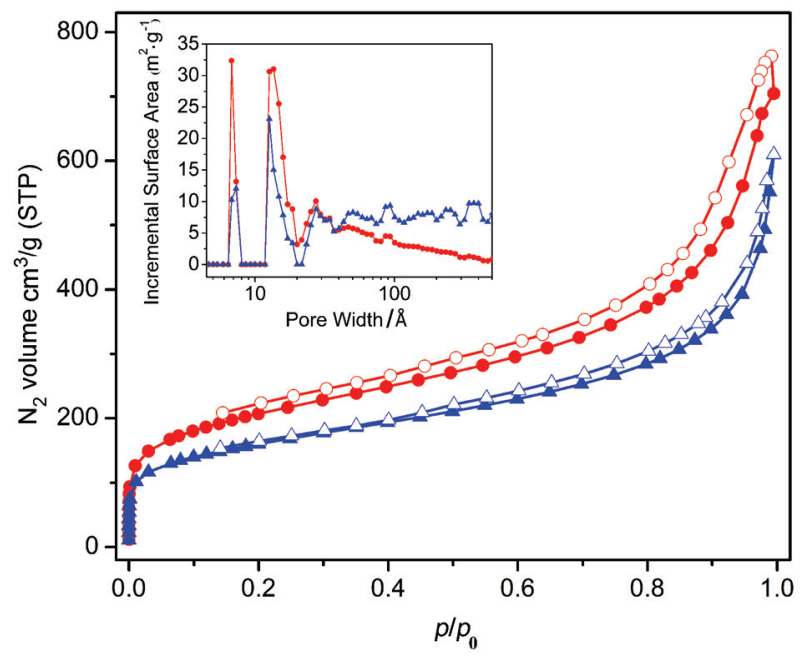

图 $6 \mathrm{~N}_{2}$ 吸附-脱附等温线 (实心为吸附曲线, 空心为脱附曲线): PAF-51-1(红色圆形), PAF-51-2(蓝色三角形). 内部小图: PAF-51-1(红 色曲线)和 PAF-51-2(蓝色曲线)的孔径分布

Figure $6 \mathrm{~N}_{2}$ adsorption (filled symbols)-desorption (empty symbols) isotherms of PAF-51-1 (red circle), and PAF-51-2 (blue triangle). Insert: the pore size distribution of PAF-51-1 (red), and PAF-51-2 (blue)
$20 \AA$ 以上的孔径分布较少. 而反应温度为 $550{ }^{\circ} \mathrm{C}$ 的 PAF-51-2 在 2 20 \& 区域孔径分布明显低于 PAF-51-1, 并在 20 500 $\AA$ 的介孔区也有一定的分布，这种现象有 可能是较高的反应温度导致 PAF-51-2 材料孔道微孔区 的局部塌陷造成的.

\section{$2.5 \mathrm{CO}_{2}$ 选择性吸附分析}

我们对 PAF-51 材料进行了 $\mathrm{CO}_{2}$ 的吸附表征, 在 $1.01 \mathrm{MPa}$ 的条件下, 分别研究了材料在 273 和 $298 \mathrm{~K}$ 对 $\mathrm{CO}_{2}$ 的吸附性质. 图中等温线初始阶段的急剧上升, 表 明 PAFs 材料孔道结构与 $\mathrm{CO}_{2}$ 分子之间可能存在有利的 分子间相互作用. 因此在高压区，我们获得了更高的 $\mathrm{CO}_{2}$ 吸附量. 从图谱中曲线的可逆性可以看出，材料有 益于捕获 $\mathrm{CO}_{2}$ 后的释放, 在实际应用中有利于循环利 用. 如表 1 所示, PAF-51-1 在 273 和 $298 \mathrm{~K}$ 条件下的总 吸附量分别为 $7.8 \mathrm{wt} \%$ 和 $3.7 \mathrm{wt} \%$. PAF-51-2 在 $273 \mathrm{~K}$ 和 $298 \mathrm{~K}$ 条件下的总吸附量分别为 $7.1 \mathrm{wt} \%$ 和 $4.6 \mathrm{wt} \%$.

表 1 PAF-51 材料的 $\mathrm{CO}_{2}$ 吸附及分离指数 Table $1 \quad \mathrm{CO}_{2}$ uptake and selectivity of PAF-51

\begin{tabular}{lcccccccc}
\hline \multirow{2}{*}{ Sample } & \multicolumn{2}{c}{$\mathrm{CO}_{2}$ uptake/wt\% } & & \multicolumn{4}{c}{$\mathrm{CO}_{2}$ selectivity $^{a}$} \\
\cline { 2 - 5 } \cline { 5 - 8 } & & & & & \multicolumn{2}{c}{$\mathrm{CO}_{2} / \mathrm{N}_{2}$} & & \multicolumn{2}{c}{$\mathrm{CO}_{2} / \mathrm{CH}_{4}$} \\
\hline PAF-51-1 & 7.8 & 3.7 & & 34.1 & 32.4 & & 8.0 & 7.3 \\
PAF-51-2 & 7.1 & 4.6 & & 52.2 & 43.6 & & 10.3 & 6.1 \\
\hline
\end{tabular}

${ }^{a}$ Calculated by the Henry's Law constants in the linear low pressure $\left(<10^{4} \mathrm{~Pa}\right)$ range.

由于 PAF-51 材料中三嗪环的存在, 导致其骨架具 有一定的富氮结构, 可提供富电子的环境, 因此可推测 这一结构使 $\mathrm{CO}_{2}$ 分子与 PAFs 材料的分子间作用力增强. 如图 7, $\mathrm{CO}_{2}$ 吸附明显优于其他气体如 $\mathrm{N}_{2}, \mathrm{CH}_{4}$. 通过初 始斜率计算其相对分离指数(表 1), PAF-51-1 在 $273 \mathrm{~K}$ 条 件下 $\mathrm{CO}_{2} / \mathrm{N}_{2}$ 的分离指数高达 34.1 , 即使在室温条件下, 其分离指数也只有微小的降低(从 34.1 降至 32.4). 引人 注目的是, PAF-51-2 分离指数高达 52.2, 对于实际应用 中从大气中分离 $\mathrm{CO}_{2}$ 具有十分积极的意义. 此分离指数 明显高于其他一些多孔材料, 如 $\mathrm{MOPs}^{[24]}, \mathrm{MOFs}^{[25]}$, BINOL-HCP ${ }^{[26]}$ ，后修饰的多孔碳材料 ${ }^{[27]}$ 等. 并在室温 条件下, 与多孔材料 BILP 系列材料 $\left.{ }^{[28} 30\right], \mathrm{PECONF}$ 系 列材料 ${ }^{[31]}$ 的分离指数具有可比性. 相似地, $\mathrm{CO}_{2} / \mathrm{CH}_{4}$ 的 分离指数也能达到 10.3 , 对于清洁再生能源的探索也有 一定的前景.

\section{3 结论}

综上所述，我们通过离子热反应成功地制备了富氮 结构的 PAF-51 材料, 并通过温度的调节得到了合适的 孔尺寸. PAF-51 材料具有极好的稳定性, 较大的比表面 积 $\left(720\right.$ 和 $\left.557 \mathrm{~m}^{2} \cdot \mathrm{g}^{-1}\right)$, 并具有良好的 $\mathrm{CO}_{2}$ 吸附能力. 此 

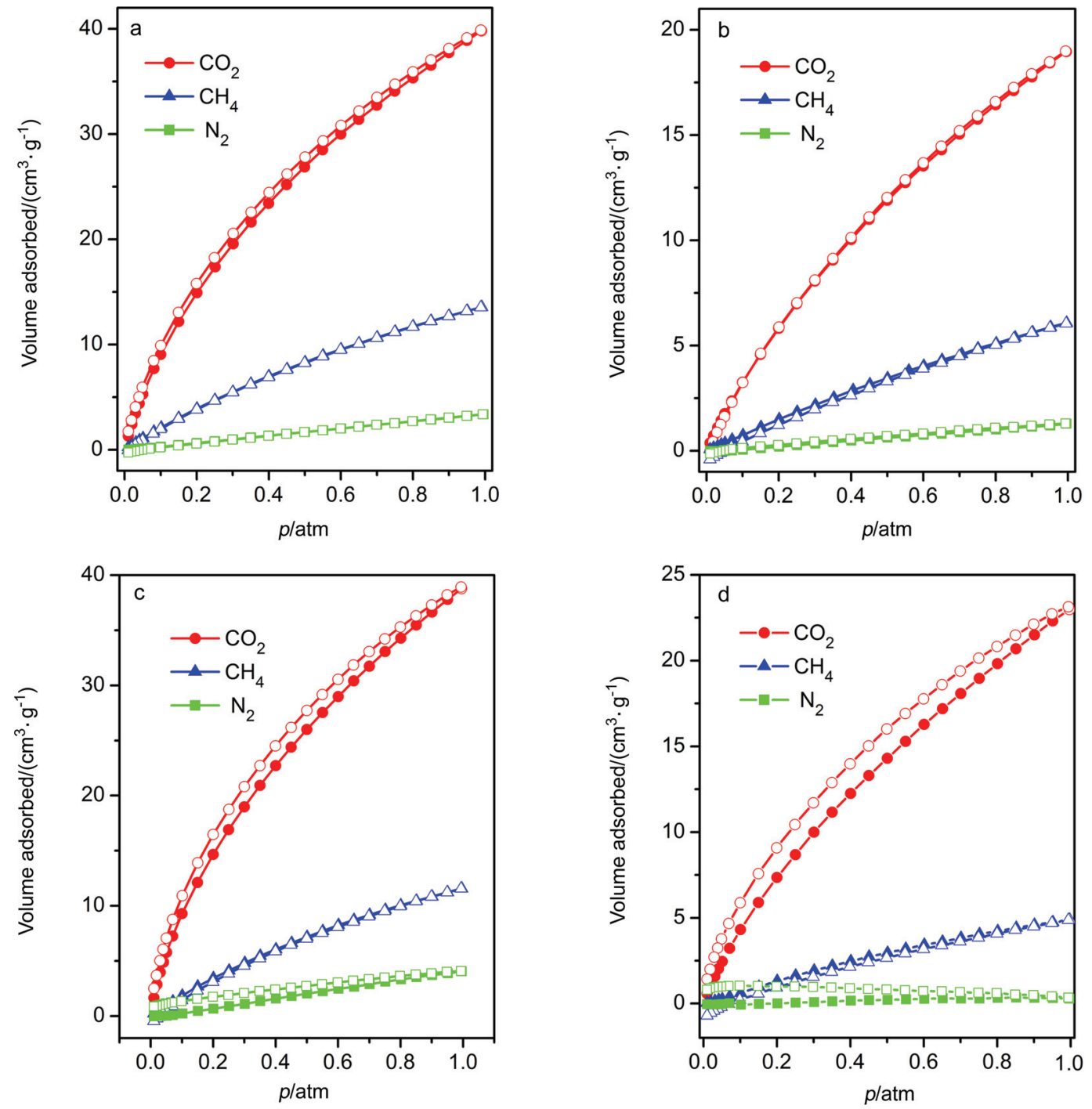

图 7 PAF-51-1 在 $273 \mathrm{~K}$ (a) 和 $298 \mathrm{~K}$ (b) 条件下的 $\mathrm{CO}_{2}$ 吸附(红色圆形), $\mathrm{CH}_{4}$ 吸附(蓝色三角形)和 $\mathrm{N}_{2}$ 吸附(绿色方形); PAF-51-2 在 $273 \mathrm{~K}$ (c) 和 $298 \mathrm{~K}$ (d) 条件下的 $\mathrm{CO}_{2}$ 吸附 (红色圆形), $\mathrm{CH}_{4}$ 吸附(蓝色三角形) 和 $\mathrm{N}_{2}$ 吸附(绿色方形)

Figure 7 The $\mathrm{CO}_{2}$ (red circle), $\mathrm{CH}_{4}$ (blue triangle), and $\mathrm{N}_{2}$ (green square) sorption isotherms of PAF-51-1 measured at $273 \mathrm{~K}$ (a) and $298 \mathrm{~K}$ (b), and PAF-51-2 measured at $273 \mathrm{~K}$ (c) and $298 \mathrm{~K}$ (d)

外, $\mathrm{CO}_{2} / \mathrm{N}_{2}$ 的分离指数在 $273 \mathrm{~K}$ 下高达 52.2 , 在 $298 \mathrm{~K}$ 下也几乎没有大幅的降低, 使 PAF-51 具有了相当可观 的 $\mathrm{CO}_{2}$ 分离性能.

\section{4 实验部分}

\section{1 四(4-氰基联苯基)硅烷 TCDPSi 的制备}

在氮气气氛下, 将四(4-溴苯基)硅烷 ${ }^{[32]}(650 \mathrm{mg}, 1$ $\mathrm{mmol}$ )与 4-氰基苯硼酸 $(588 \mathrm{mg}, 4 \mathrm{mmol}), \mathrm{Na}_{2} \mathrm{CO}_{3}(4 \mathrm{~g})$ 的 混合物溶于甲苯 $(50 \mathrm{~mL})$ 中, 加入去离子水 $(20 \mathrm{~mL})$, 乙 醇 $(20 \mathrm{~mL})$, 及 $\mathrm{Pd}\left(\mathrm{PPh}_{3}\right)_{4}(500 \mathrm{mg})$. 溶液在 $90{ }^{\circ} \mathrm{C}$ 搅拌 3 $\mathrm{d}$, 减压除去挥发性溶剂, 用二氯甲烷萃取剩余产物后 浓缩成固体, 用柱层析法进一步提纯, 得到白色固体粉 末，在 $80{ }^{\circ} \mathrm{C}$ 真空干燥 $2 \mathrm{~h}$.

\subsection{PAF-51 的制备}

在手套箱中, 将单体 TCDPSi 与无水氯化锌通过仔 细研磨之后, 共同转移至安瓿中. 安瓿维持真空密封状 态, 分别在 400 和 $550{ }^{\circ} \mathrm{C}$ 条件下反应 $40 \mathrm{~h}$. 待冷却至室 温后, 小心打开安瓿, 将得到的黑色固体在去离子水中 搅拌 $48 \mathrm{~h}$, 以去除反应中的氯化锌. 所得沉淀在 $1 \mathrm{~mol} / \mathrm{L}$ 盐酸中继续傥拌过夜, 以进一步提纯. 最后用无水四氢 呋喃交换出客体水分子，在 $100{ }^{\circ} \mathrm{C}$ 条件下真空干燥 $6 \mathrm{~h}$.

\section{References}

[1] Haszeldine, R. S. Science 2009, 325, 1647.

[2] Leaf, D.; Verolmec, H. J. H.; Hunt, W. F. Jr. Environ. Int. 2003, 29, 303.

[3] Rochelle, G. T. Science 2009, 325, 1652

[4] Vallee, G.; Mougine, P.; Julian, S.; Furst, W. Ind. Eng. Chem. Res. 1999, 38,3473 
[5] Lozano-Castello, D.; Cazorla-Amoros, D.; Linares-Solano, A. Carbon 2004, 42, 1233.

[6] Demessence, A.; D'Alessandro, D. M.; Foo, M. L.; Long, J. R. J. Am. Chem. Soc. 2009, 131, 8784.

[7] Nakagawa, K.; Tanaka, D.; Horike, S.; Shimomura, S.; Higuchi, M.; Kitagawa, S. Chem. Commun. 2010, 46, 4258.

[8] Sumida, K.; Rogow, D. L.; Mason, J. A.; McDonald, T. M.; Bloch, E. D.; Herm, Z. R.; Bae, T. H.; Long, J. R. Chem. Rev. 2012, 112, 724.

[9] Lee, J. Y.; Wood, C. D.; Bradshaw, D.; Rosseinsky, M. J.; Cooper, A. I. Chem. Commun. 2006, 25, 2670.

[10] Ghanem, B. S.; McKeown, N. B.; Budd, P. M.; Al-Harbi, N. M.; Fritsch, D.; Heinrich, K.; Starannikova, L.; Tokarev, A.; Yampolskii, Y. Macromolecules 2009, 42, 7881.

[11] McKeown, N. B.; Budd, P. M. Macromolecules 2010, 43, 5163.

[12] Dawson, R.; Adams, D. J.; Cooper, A. I. Chem. Sci. 2011, 2, 1173.

[13] Kuhn, P.; Antonietti, M.; Thomas, A. Angew. Chem., Int. Ed. 2008, $47,3450$.

[14] Kuhn, P.; Thomas, A.; Antonietti, M. Macromolecules 2009, 42, 319.

[15] Bojdys, M. J.; Jeromenok, J.; Thomas, A.; Antonietti, M. Adv. Mater. 2010, 22, 2202.

[16] Yuan, D.; Lu, W.; Zhao, D.; Zhou, H.-C. Adv. Mater. 2011, 23, 3723.

[17] Lu, W.-G.; Yuan, D.-Q.; Zhao, D.; Schilling, C. I.; Plietzsch, O.; Muller, T.; Brase, S.; Guenther, J.; Blumel, J.; Krishna, R.; Li, Z.; Zhou, H.-C. Chem. Mater. 2010, 22, 5964.

[18] Ren, H.; Ben, T.; Wang, E.-S.; Jing, X.-F.; Xue, M.; Liu, B.-B.; Cui, Y.; Qiu, S.-L.; Zhu, G.-S. Chem. Commun. 2010, 46, 291.
[19] Ren, H.; Ben, T.; Sun, F.-X.; Guo, M.-Y.; Jing, X.-F.; Ma, H.-P.; Cai, K.; Qiu, S.-L.; Zhu, G.-S. J. Mater. Chem. 2011, 21, 10348.

[20] Yuan, Y.; Yan, Z.-J.; Ren, H.; Liu, Q.-Y.; Zhu, G.-S.; Sun, F.-X. Acta Chim. Sinica 2012, 70, 1446. (元野，间卓君，任浩，刘青英，朱广 山, 孙福兴, 化学学报, 2012, 70, 1446.)

[21] Zhang, T.-T.; Wang, H.-T.; Ma, H.-P.; Sun, F.-X.; Cui, X.-Q.; Zhu. G.-S. Acta Chim. Sinica 2013, 71, 1598. (张婷婷，王海涛，马和平, 孙福兴, 崔小强, 朱广山, 化学学报, 2013, 71, 1598.)

[22] Wang, W.; Ren, H.; Sun, F. X.; Cai, K.; Ma, H. P.; Du, J.-S.; Zhao, H.-J.; Zhu, G.-S. Dalton Trans. 2012, 41, 3933.

[23] Patel, H. A.; Karadas, F.; Canlier, A.; Park, J.; Deniz, E.; Jung, Y.; Atilhan, M.; Yavuz, C. T. J. Mater. Chem. 2012, 22, 8431.

[24] Dawson, R.; Stöckel, E.; Holst J. R.; Adams, D. J.; Cooper, A. I. Energy Environ Sci. 2011, 4, 4239.

[25] Liang, Z.; Du, J.; Sun, L.; Xu, J.; Mu, Y.; Li, Y.; Yu, J.; Xu, R. Inorg. Chem. 2013, 52, 10720.

[26] Dawson, R.; Stevens, L. A.; Drage, T. C.; Snape, C. E.; Smith, M. W.; Adams, D. J. Am. Chem. Soc. 2012, 134, 10741.

[27] Wang, J.; Heerwig, A.; Lohe, M. R.; Oschatz, M.; Borchardt, L.; Kaskel, S. J. Mater. Chem. 2012, 22, 13911.

[28] Rabbani, M. G.; El-Kaderi, H. M. Chem. Mater. 2011, 23, 1650.

[29] Rabbani, M. G.; El-Kaderi, H. M. Chem. Mater. 2012, 24, 1511.

[30] Gulam, R. M.; Reich, T. E.; Kassab, R.; Jackson, K. T.; El-Kaderi, H. M. Chem. Commun. 2012, 48, 1141.

[31] Mohanty, P.; Kull, L. D.; Landskron, K. Nature Commun. 2011, 2, 401.

[32] Liu, F. Q.; Tilley, T. D. Inorg. Chem. 1997, 36, 5090.

(Qin, X.) 